\title{
Latitude distribution of nonradial pulsations in rapidly rotating $B$ stars
}

\author{
S. Jankov ${ }^{1,2,3}$, P. Mathias ${ }^{1}$, A. Domiciano de Souza Jr. ${ }^{2}$, \\ K. Uytterhoeven ${ }^{4}$, C. Aerts ${ }^{4}$ \\ ${ }^{1}$ Observatoire de la Côte d'Azur, UMR 6528, F-06304 Nice, France \\ ${ }^{2}$ LUAN, UMR 6525, U.N.S.A., Parc Valrose, F-06108, Nice, France \\ ${ }^{3}$ Astronomical Observatory, MNTRS 1940, 11050 Beograd, Serbia \\ ${ }^{4}$ Institute of Astronomy, Katholieke Universiteit Leuven, \\ Celestijnenlaan 200B, B-3001 Leuven, Belgium
}

\begin{abstract}
We present a method for the analysis of latitude distribution associated with temperature and/or velocity perturbations of the stellar surface due to non-radial pulsation (NRP) modes in rapidly rotating B stars. The technique is applied together with Fourier Doppler Imaging (FDI) to high resolution and high signal-to-noise ratio spectroscopic observations of $\epsilon$ Per. The main advantage of this approach is that it decomposes complex multi-periodic line profile variations into single components, allowing the detailed analysis of each mode separately. We study the $10.6-\mathrm{d}^{-1}$ frequency that is particularly important for modal analysis of non-radial pulsations in the star.
\end{abstract}

\section{Introduction}

In rapid rotating stars there is a one-to-one mapping between the points in the broadened line profile and the position of perturbations on the stellar surface (Vogt \& Penrod 1983). The technique of the so-called "Doppler mapping" led to various methods to identify pulsation parameters (order $m$ and degree $\ell$ ) from high-resolution, high signal-to-noise spectroscopic observations (see e.g. Telting $\&$ Schrijvers 1997). However, no attempts have been made to determine the latitude $b$ of the maximum of the pulsation amplitude at the stellar surface. In this paper we show how this parameter can be evaluated from spectroscopic observations and we discuss how it can improve the results of classical modal analysis.

\section{The method}

A photospheric absorption line in which rotation is the dominant broadening mechanism displays time-variable perturbations ("bumps" or "dips") which are carried (as the star rotates) across the stellar disc, causing the bumps to change their Doppler shifts in accordance with their projected distances from the stellar 


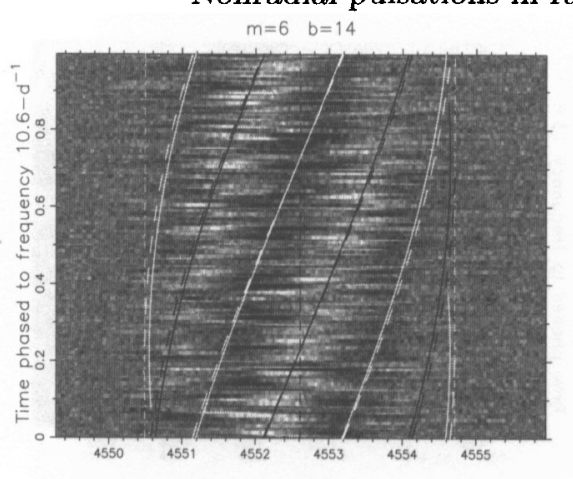

Frequency $10.6-d^{-1}$
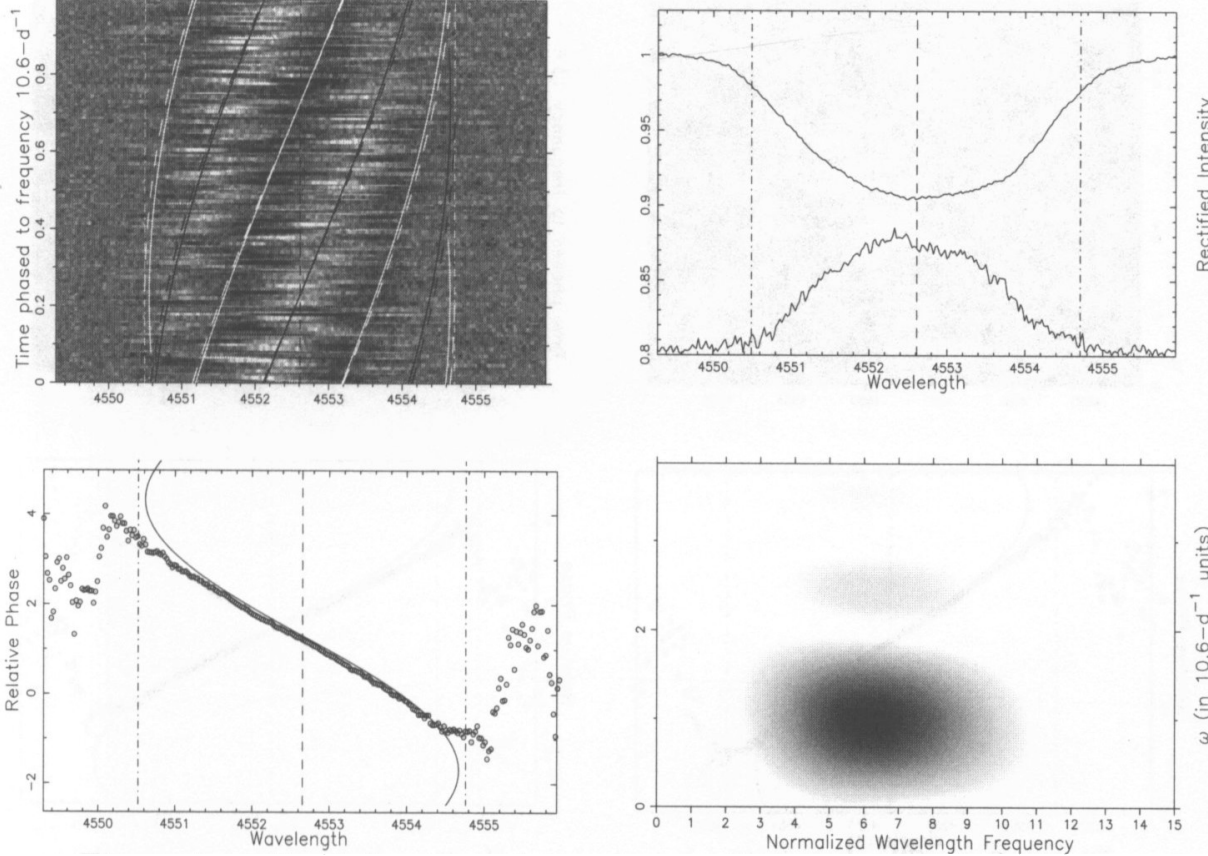

Figure 1. Top-left: dynamic spectrum. Bottom-left: NRP phases.

Bottom-right: FDI. Top-right: NRP amplitudes and average profile.

rotation axis which depends on azimuth $\phi$ :

$$
\Delta \lambda=\frac{\lambda}{\mathrm{c}} V_{\mathrm{e}} \sin i \cos b \sin \phi
$$

where the projected rotational velocity $V_{\mathrm{e}} \sin i$ is known, for example, from Fourier analysis of the average profile. Relating the azimuth to time $t$ through: $\phi=\frac{\omega}{m} t$, the bump paths, corresponding to the observed frequency $\omega$, can be traced in the dynamic (wavelength/time) spectrum. In addition, the NRP phase $\Phi$ can be measured by fitting the sinusoids in each wavelength bin of the dynamic spectrum and the phase diagrams (wavelength $/ \Phi$ ) can be used to determine $b$, relating $\phi$ (in Eq. 1) to $\Phi$ through: $\phi=\frac{\Phi}{m} 2 \pi$. From the above equations it is obvious that the bump paths, as well as NRP phase, trace out a sinusoidal variation with an amplitude equal to $V_{\mathrm{e}} \sin i \cos b$, from which $b$ can be deduced if $V_{\mathrm{e}} \sin i$ is known. This value of $b$ depends also on $m$, and should be consistent with $l$ that can be evaluated using other methods (e.g. Fourier Doppler Imaging).

\section{The application to the $10.6-\mathrm{d}^{-1}$ frequency of $\epsilon$ Per}

We use the spectroscopic data set of $\epsilon$ Per, for which observations and reduction procedure have been described elsewhere (De Cat et al. 2000), applying the method to the $10.6-\mathrm{d}^{-1}$ frequency. For modal analysis it is very important 

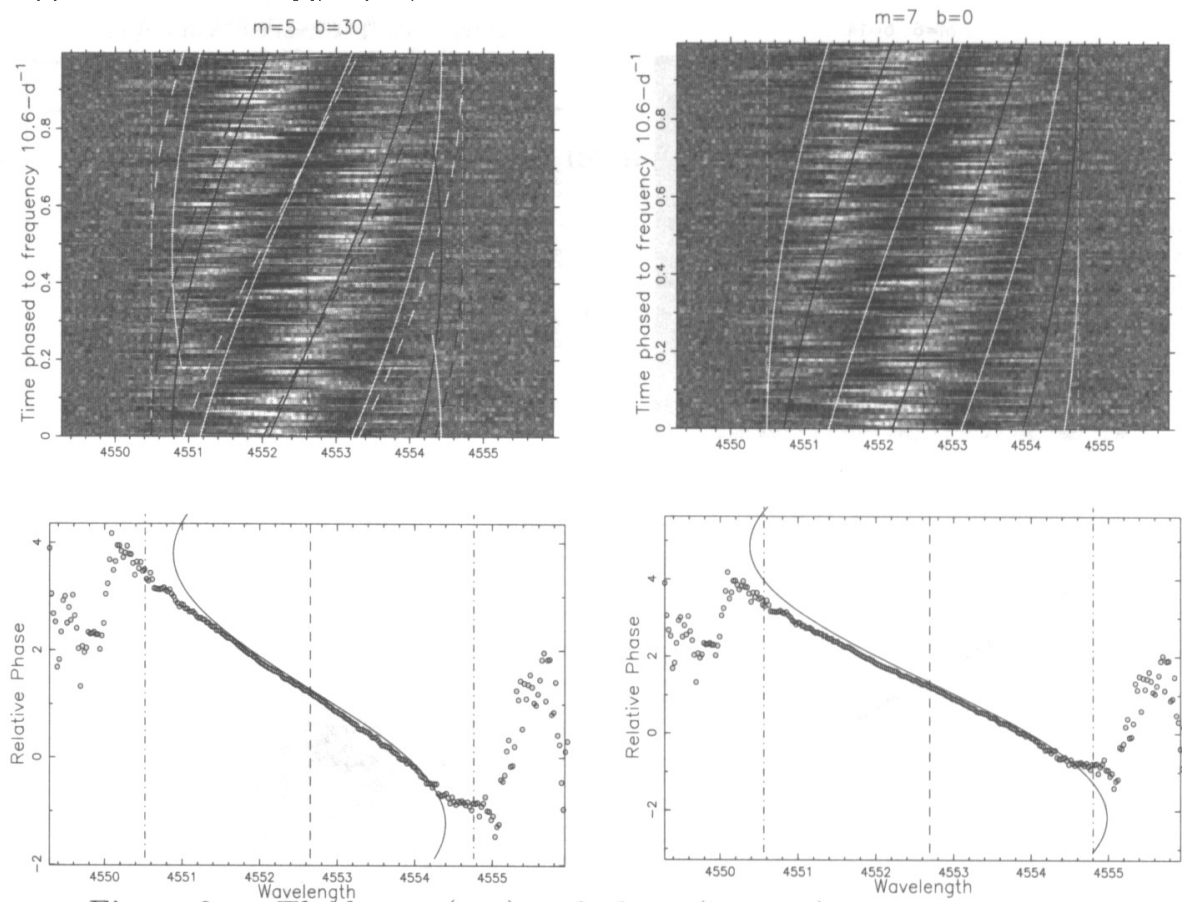

Figure 2. The bump (top) and phase (bottom) paths corresponding to a mode $|m|=5$ (left) and $|m|=7$ (right).

to study whether this frequency corresponds to an independent mode in the star or it is just a harmonic of the 5.6- $\mathrm{d}^{-1}$ frequency (Telting \& Schrijvers 1997). Although the star's pulsation is multi-periodic and complex, the lineprofile variabilities caused by the individual pulsation modes were separated in frequency by Fourier analysis which allows the characteristics of each mode to be studied separately. The dynamic spectrum of residuals (average spectrum subtracted) is presented in the Fig. 1 (top-left) where the bump paths correspond to a $|m|=6$ mode confined to the equator (dashed lines) or to the latitude $b=14^{\circ}$ (full lines). At the bottom-left the measured NRP phases are plotted as circles, while the full line represents the sinusoidal fit which, for $V_{\mathrm{e}} \sin i=$ $139 \mathrm{~km} \mathrm{~s}^{-1}$, corresponds to $b=14^{\circ}$. The Fourier-Doppler diagram is plotted at the bottom-right of the figure, while the measured NRP amplitudes and the average profile are presented at the top-right. Fig. 2 shows the bump (top) and phase (bottom) paths corresponding to a mode $|m|=5$ (left) and $|m|=7$ (right). The full lines correspond to $b=30^{\circ}(|m|=5)$ and $b=0^{\circ}(|m|=7)$, and dashed lines correspond to the equator.

\subsection{Discussion and conclusions}

Since the normalized wavelength frequency in FDI diagrams is an indicator of the degree $\ell$, (e.g. Telting \& Schrijvers 1997), our analysis implies $\ell=6 \pm 1$. So, we explored the parameter space only for $|m|=6 \pm 1$ that produced better fits than $|m|<5$. At the $10.6-\mathrm{d}^{-1}$ frequency our observations are consistent 


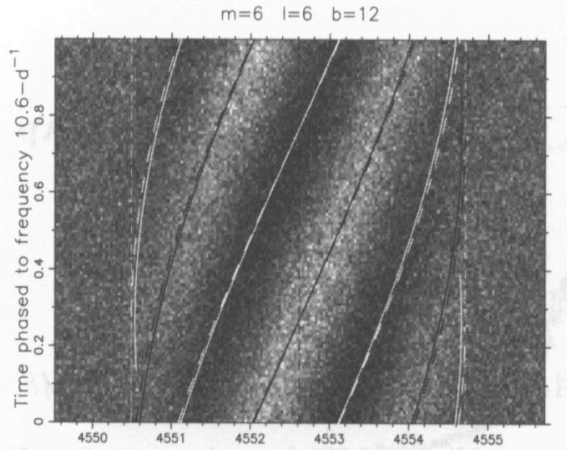

Frequency $10.6-d^{-1}$
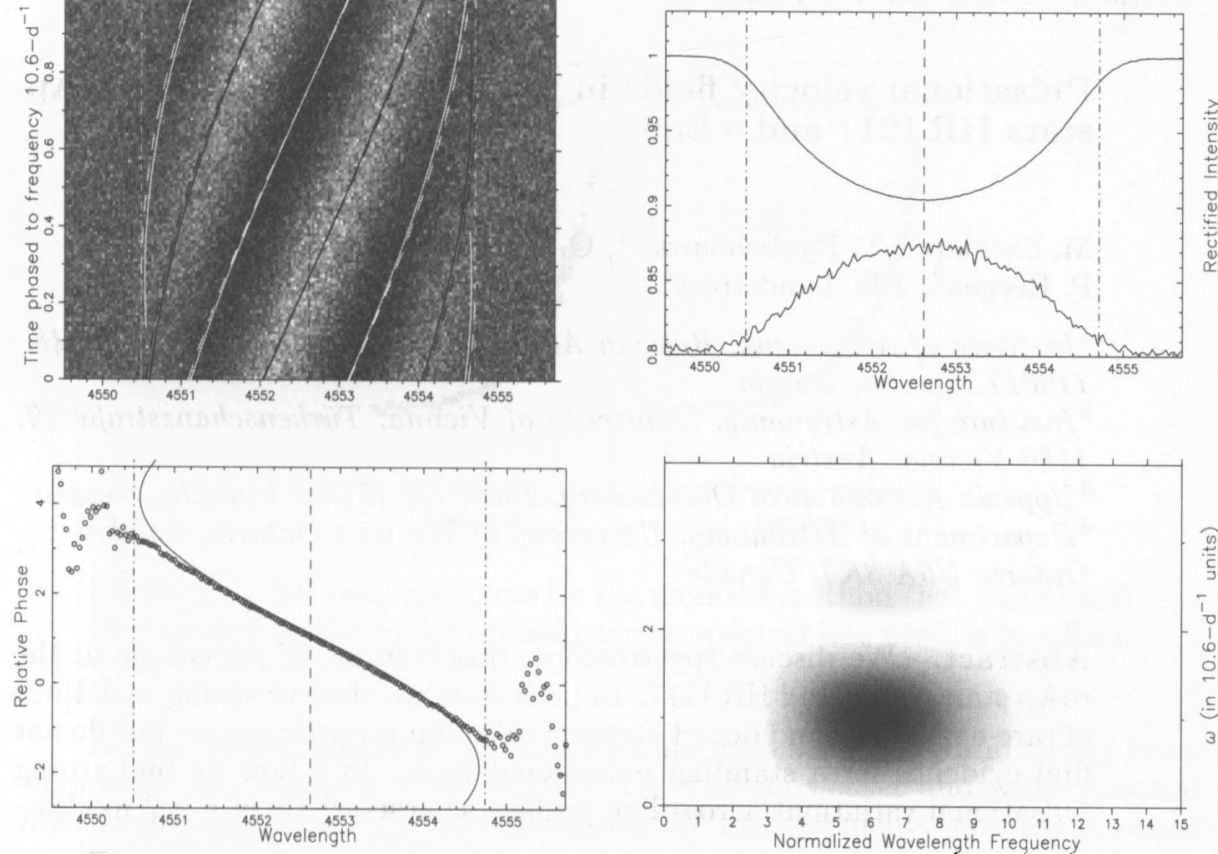

Figure 3. Same as in Fig. 1 but for the noisy synthetic spectra of the $|m|=6, l=6$ mode in a star tilted at $i=45^{\circ}$.

with an independent $|m|=6, l=6$ mode, although the associated apparent latitude is $b=14^{\circ}$. In fact, the latitude should be corrected for the projection effect: for a tilted star the maximum of pulsation is shifted toward the subobserver latitude. The analysis of generated noisy spectra for $|m|=6, \ell=6$ mode in a star tilted at $i=45^{\circ}$ (Fig. 3) implies $b=12^{\circ}$ for $|m|=6$, and $\ell=6$. When compared to results from Fig. 1, the similarity of diagrams is obvious. The $|m|=5, \ell=6$ mode is also consistent with deduced $b=30^{\circ}$ (although the similarity of synthetic spectra is not as good as for $|m|=6, \ell=6$ ), while the $|m|=7$ modes should be excluded. Limits are depicted as vertical dashed side-lines, implying $\cos b>1$.

\section{References}

De Cat, P., Telting, J., Aerts, C., Mathias, P. 2000, A\&A, 359, 539

Telting, J.H., Schrijvers, C. 1997, A\&A, 317, 723

Vogt, S.S., Penrod, G.D. 1983, ApJ, 275, 661 